\title{
Fluorodeoxyglucose positron emission tomography improves preoperative staging of resectable lung metastasis
}
U. Pastorino, $M D^{\mathrm{a}}$
G. Veronesi, $M D^{\mathrm{b}}$
C. Landoni, $\mathrm{MD}^{\mathrm{C}}$
M. Leon, $\mathrm{PhD}^{\mathrm{d}}$
M. Picchio, $M D^{\mathrm{c}}$
P. G. Solli, $M D^{\mathrm{a}}$
F. Leo, MD ${ }^{b}$
L. Spaggiari, $M D^{\mathrm{b}}$
G. Pelosi, MD
M. Bellomi, $M D^{f}$
F. Fazio, Professor ${ }^{\mathrm{c}}$

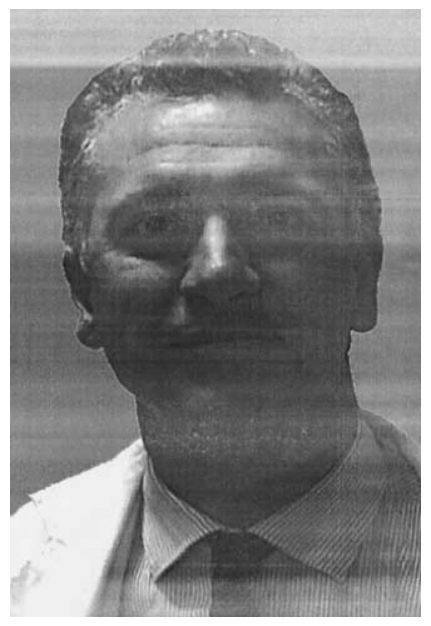

Dr Pastorino

\footnotetext{
From the Department of Thoracic Surgery, Istituto Nazionale Tumori ${ }^{\mathrm{a}}$; the Department of Thoracic Surgery, European Institute of Oncology ; INB CNR, University of Milano Bicocca ${ }^{c}$; Division of Statistic, European Institute of Oncology ${ }^{\mathrm{d}}$; Department of Pathology, European Institute of Oncolo$\mathrm{gy}^{\mathrm{e}}$; and Division of Radiology, European Institute of Oncology, ${ }^{\mathrm{f}}$ Milan, Italy.

Received for publication June 22, 2002; revisions requested Sept 11, 2002; revisions received Nov 27, 2002; accepted for publication Jan 22, 2003

Address for reprints: Ugo Pastorino, MD, Department of Thoracic Surgery, Istituto Nazionale Tumori, Via Venezian 1, 20133 Milan, Italy (E-mail: ugo.pastorino@ istitutotumori.mi.it).

J Thorac Cardiovasc Surg 2003;126: 1906-10

Copyright () 2003 by The American Association for Thoracic Surgery

$0022-5223 / 2003 \$ 30.00+0$

doi:10.1016/S0022-5223(03)00211-3
}

Objective: F-18 fluorodeoxyglucose positron emission tomography (FDG-PET) is now a procedure of proven clinical value in the staging of primary lung cancer. This study evaluated the role of PET in the preoperative assessment of resectable lung metastases.

Methods: Eighty-six patients with previously treated malignancy and proven or suspected lung metastases, deemed resectable at computed tomography scan, were investigated with 89 preoperative PET procedures. Primary tumor sites were: gastrointestinal in 32 cases, sarcoma in 13, urologic in 14, breast in 8 , head and neck in 7 , gynecologic in 5, thymus in 5, other in 5. Seventy lung resections were performed in 68 patients of whom only 54 proved to be lung metastasis, 7 were primary lung tumors, and 9 were benign lesions.

Results: In 19 cases (21\%) lung surgery was excluded on the basis of PET scan results due to extrapulmonary metastases (11 cases), primary site recurrence (2), mediastinal adenopathy (2), or benign disease (4). All mediastinal node metastases (7 cases) were detected by PET with a sensitivity, accuracy, and negative predictive value for mediastinal staging of $100 \%, 96 \%$, and $100 \%$, respectively, versus $71 \%$, $92 \%$, and $95 \%$ of the computed tomography scan. In the group of patients who underwent lung resection, PET sensitivity for detection of lung metastasis was $87 \%$.

Conclusions: PET scan proved to be a valuable staging procedure in patients with clinically resectable lung metastasis and changed the therapeutic management in a high proportion of cases.

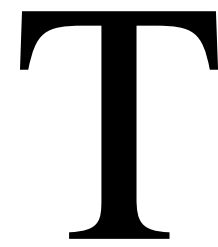

he surgical treatment of isolated pulmonary metastases has become a widely accepted procedure, with low morbidity and mortality. ${ }^{1,2}$ The oncologic criteria for selection of surgical candidates and the main factors affecting prognosis were investigated in large series of cases. ${ }^{3,4}$ Nonetheless, only $30 \%$ of patients with isolated and anatomically resectable lung metastases can be cured by surgery, and there is a need to improve the preoperative selection for optimal candidates.

The value of positron emission tomography (PET) in preoperative staging has been clearly demonstrated for primary lung cancer, ${ }^{5-8}$ where PET assessment of mediastinal and extrathoracic occult metastasis avoids unnecessary surgery in about $10 \%$ of patients. 
TABLE 1. Primary site tumor in 89 cases of PET scan performed in 86 patients with suspected lung metastases divided for pathological results in case of lung resection (lung metastasis, second primary tumor, and benign lesion) and for causes of exclusion from surgery (distant metastasis, local recurrence, mediastinal node metastases, and benign lung lesions)

\begin{tabular}{|c|c|c|c|c|c|c|c|c|}
\hline & \multicolumn{4}{|c|}{ Surgery } & \multicolumn{4}{|c|}{ No surgery } \\
\hline & Total & LM & SPT & Benign & DM & LR & Med & Benign \\
\hline Overall & 89 & 54 & 7 & 9 & 11 & 2 & 2 & 4 \\
\hline \multicolumn{9}{|l|}{ Primary site } \\
\hline Colorectal & 32 & 24 & 1 & 3 & 3 & 1 & - & - \\
\hline Sarcoma & 17 & 12 & - & - & 3 & 1 & 1 & - \\
\hline Urologic & 14 & 7 & 2 & - & 2 & - & 1 & 1 \\
\hline Breast & 6 & 2 & 2 & 1 & 1 & - & - & - \\
\hline Head/neck & 7 & 3 & 1 & 1 & - & - & - & 1 \\
\hline Gyn & 4 & 2 & - & 1 & 1 & - & - & - \\
\hline Thymus & 5 & 3 & - & - & 1 & - & - & 1 \\
\hline Testis & 2 & 1 & - & 1 & - & - & - & - \\
\hline Skin/Mel & 3 & - & 1 & 1 & - & - & - & 1 \\
\hline Teratoma & 1 & - & - & 1 & - & - & - & - \\
\hline
\end{tabular}

$L M$, Confirmed lung metastasis; SPT, second primary tumor; $D M$, distant metastasis; $L R$, local recurrence; $M E D$, mediastinal node metastasis; Gyn, gynecologic tumor; Mel, melanoma.

Presence of extrathoracic disease in patients with lung metastasis including primary site relapse represents, with rare exceptions, the main reason for exclusion from surgical treatment. Recently, mediastinal lymph node staging has been suggested as another important prognostic factor in otherwise resectable lung metastases. ${ }^{3,9,10}$ In this view, accurate staging of both extrathoracic deposits and mediastinal nodes becomes essential to improve selection of surgical candidates.

Preoperative PET can modify the clinical management of patients with lung metastasis by detecting occult metastases in other organs, primary site recurrence, nodal spread at the hilar or mediastinal level, as well as by differentiating malignant from benign pulmonary nodules. PET can also help to exclude distant metastases in patients with equivocal evidence at computed tomography (CT) scan or ultrasound. The aim of this study was to evaluate the clinical value of F-18 fluorodeoxyglucose (FDG) PET in addition to spiral $\mathrm{CT}$ for preoperative staging of lung metastasis.

\section{Patients and Methods}

\section{Patients}

Patients with previously treated malignancy and proven or suspected lung metastases deemed resectable at CT scan were evaluated with FDG-PET. A total of 86 patients were studied with 89 PET procedures between August 1998 and December 2000. There were 48 men and 38 women with a mean age of 60 years. Median disease-free survival was 24 months. Primary tumor site of all patients is presented in Table 1 . Thirty patients (35\%) had previously submitted to lung metastasectomy and presented recurrent lung metastasis. All patients were evaluated with contrast spiral CT of the chest and abdomen and additional imaging tests, such as nuclear magnetic resonance imaging or ultrasound, in case of symptoms or signs suggestive of distant metastasis or primary site recurrence. Resectability was based on the following criteria: primary tumor previously cured or curable, no evidence of extrapulmonary metastases, complete removal of all pulmonary metastases feasible, planned resection volume tolerable by the patient.

Among patients who underwent resection for lung metastases, 7 had received preoperative chemotherapy within 3 months of metastasectomy.

\section{PET}

Images were acquired with a General Electric Advance PET scanner (General Electric Medical Systems, Milwaukee, Wis) 1 hour after injection of approximately $370 \mathrm{MBq}$ of F-18 FDG. Each patient was imaged with arms over the head, covering a field of view from neck to pelvis. A transmission scan was then performed on the thorax region to measure attenuation. Raw data were corrected for measured attenuation by segmented transmission data and then reconstructed in transaxial images by an iterative algorithm with 16 subset and order 4. Parametric standard uptake value (SUV) transaxial images were obtained as follows: SUV = (pixelby-pixel activity in becquerels per cubic centimeter)/(injected dose in megabecquerls per body weight in kilograms). Circular regions of interest (ROIs) were manually drawn on transaxial images around the focal FDG-uptake area. No correction for partialvolume effect was performed. Mean value of maximum SUV of different slices of each nodule was considered for statistical analysis. Data on SUV were available for 70 of 89 PET procedures.

\section{Surgery and Pathologic Results}

Seventy lung resections were performed in 68 patients with the following pathologic results: 54 lung metastases, 7 primary lung tumors, and 9 benign lesions. Of 54 patients with lung metastasis, 41 had monolateral lung resection and 13 bilateral resection. A single lung metastasis was present in 26 patients, from 2 to 5 metastases in 21 patients, and more than 5 in 7 patients. A proper lymph node sampling was performed in 31 of 54 patients with proven lung metastasis: this included an average of 12.2 nodes and at least 1 hilar and 1 mediastinal station. In an additional 19 
TABLE 2. Results of PET and CT detection of hilar and mediastinal node metastasis in 50 patients with lung metastasis submitted to lymph node sampling (31) or follow-up study (19)

\begin{tabular}{|c|c|c|c|c|c|c|c|c|c|c|}
\hline & $\begin{array}{c}\text { No. of } \\
\text { patients }\end{array}$ & TP & FN & TN & FP & Sens (\%) & Spec (\%) & Accur (\%) & PPV (\%) & NPP (\%) \\
\hline \multicolumn{11}{|l|}{ PET } \\
\hline Med & 50 & 7 & 0 & 41 & 2 & 100 & 95 & 96 & 78 & 100 \\
\hline Hil & 50 & 2 & 3 & 44 & 1 & 40 & 98 & 92 & 66 & 94 \\
\hline $\mathrm{M}$ or $\mathrm{H}$ & 50 & 8 & 3 & 36 & 3 & 72 & 92 & 88 & 72 & 92 \\
\hline \multicolumn{11}{|l|}{ CT } \\
\hline Med & 50 & 5 & 2 & 41 & 2 & 71 & 95 & 92 & 71 & 95 \\
\hline Hil & 50 & 0 & 5 & 44 & 1 & 0 & 98 & 88 & 0 & 90 \\
\hline $\mathrm{M}$ or $\mathrm{H}$ & 50 & 5 & 6 & 36 & 3 & 45 & 92 & 82 & 62 & 86 \\
\hline
\end{tabular}

$\overline{T P}$, True-positive; $F N$, false-negative; $T N$, true-negative; $F P$, false-positive; $P P V$, positive predictive value; $N P P$, negative predictive value; $M e d$, mediastinal; Hil, hilar; $M$ or $H$, mediastinal or hilar.

patients, the mediastinal involvement was assessed by follow-up data ( 8 of these had 1 hilar station pathologically evaluated, average 1.5 nodes). Nodal status was not available in 4 patients.

\section{Statistical Analysis}

Comparisons of percentage of positive nodes by histologic type and sensitivity by histologic type or by nodule size category were done by the Fisher exact test. Mean SUV values were compared taking into account nodule size by regression analysis. All analyses were done with SAS software (SAS, Inc, Cary, NC).

\section{Results}

In 19 of 89 cases (21\%) lung surgery was not performed on the basis of PET results, which showed extrapulmonary metastasis in 11 cases, primary site recurrence in 2 , mediastinal adenopathy in 2 , benign lung nodule in 4 (Table 1). Sites of local recurrences were colon cancer in 1 patient and sarcoma of the arm in another. Of the 11 cases of distant metastases, 5 bone lesions were confirmed by rib biopsy (2), magnetic resonance imaging (MRI) (2), or bone scan (1); 2 adrenal lesions were confirmed by CT; 1 chest wall metastasis with needle biopsy; 1 pancreatic metastasis by MRI; 1 liver and abdominal node metastases were confirmed by clinical follow-up only. Of the 2 mediastinal nodes, 1 was confirmed by mediastinoscopy and the other by progression of $\mathrm{N} 2$ disease at follow-up. In the 4 cases excluded from surgery, benignity was assessed by clinical follow-up, without biopsy.

Among patients submitted to lung resection (70), in 2 cases clinical management was changed by PET detection of a colon cancer recurrence that was resected before lung metastasectomy and 1 liver metastases that was treated after lung surgery. In 2 further cases, distant metastases were identified by PET in the liver and pelvis, but lung metastasectomy was performed due to negative CT scan. Later follow-up confirmed the true positivity of PET staging, with tumor progression at these distant sites. There were 3 falsenegative PET extrapulmonary sites of disease: a brain meningioma, a pleural micrometastasis (diameter 1-3 mm), and a small breast cancer. A false-positive PET at distant sites occurred in 7 patients: bone hot spots in 2 patients (spine, chest wall), site of previous surgical resection in 1 patient (pelvis), neck and abdominal lymph nodes in 2 cases, and prostatic hyperplasia in 1 case. PET sensitivity, specificity, and accuracy for detection of distant deposits were $85 \%$, $90 \%$, and $89 \%$, respectively.

Among patients subjected to lung metastasectomy, all cases $(7 / 50,14 \%)$ of mediastinal nodes involvement were detected by PET, with only 2 cases of false-positive PET at the mediastinal level. Overall sensitivity, accuracy, and negative predictive value at the mediastinal level were greater that those of CT scan $(100 \%, 96 \%$, and $100 \%$ vs $71 \%, 92 \%$, and $95 \%$, respectively, Table 2). In 2 cases of positive mediastinal PET and negative CT scan of the chest, we could have avoided exploratory thoracotomy by confirming PET results through mediastinoscopy. In the other 5 cases, preoperative detection of localized mediastinal disease by PET allowed a better approach by lung metastasectomy combined with radical mediastinal lymphadenectomy or mediastinal adjuvant radiotherapy. In 2 additional cases PET detection of major mediastinal involvement led to exclusion from surgery. At the hilar station there were 3 false-negative PET metastases (sensitivity 40\%) and 5 falsenegative CT scans (sensitivity 0\%) (Table 2). Positive lymph nodes occurred in $23 \%$ of patients with epithelial tumors (13\% mediastinal) and $17 \%$ of patients with sarcoma metastases (17\% mediastinal).

PET was positive at the pulmonary level in 47 of 54 (87\%) patients who underwent metastasectomy. Table 3 describes sensitivity of PET in relation to lung lesions size and primary tumor type. Only $40 \%$ of nodules smaller than $10 \mathrm{~mm}$ were PET positive; sensitivity increased to $90 \%$ for nodules between 10 to $20 \mathrm{~mm}$ and $94 \%$ for nodules greater than $20 \mathrm{~mm}(P=.0149)$. PET sensitivity was $92 \%$ for colorectal cancer, $86 \%$ for urologic neoplasms, $75 \%$ for sarcomas, and $50 \%$ for breast cancer. No significant association between sensitivity and tumor type was evident $(P=$ 
TABLE 3. PET sensitivity in lung metastasis

\begin{tabular}{|c|c|c|c|c|}
\hline & PET positive & PET negative & $\%$ & $P$ value* \\
\hline Overall sensitivity & 47 & 7 & 87 & \\
\hline \multicolumn{5}{|l|}{ Sensitivity by nodule size $\dagger$} \\
\hline$\leq 10 \mathrm{~mm}$ & 2 & 3 & 40 & \\
\hline $11-20 \mathrm{~mm}$ & 18 & 2 & 90 & \\
\hline$>21 \mathrm{~mm}$ & 27 & 2 & 93 & 0.0149 \\
\hline \multicolumn{5}{|l|}{ Sensitivity by histology } \\
\hline Colon & 22 & 2 & 92 & \\
\hline Urologic & 6 & 1 & 86 & \\
\hline Sarcoma & 9 & 3 & 75 & \\
\hline Head/neck & 3 & 0 & 100 & \\
\hline Thymus & 3 & 0 & 100 & \\
\hline Breast & 1 & 1 & 50 & \\
\hline Gynecologic & 2 & 0 & 100 & \\
\hline Testis & 1 & 0 & 100 & 0.51 \\
\hline
\end{tabular}

*Fisher exact extension associated $P$ value.

tMaximum nodule size was considered for calculating sensitivity in patients with multiple nodules.

.5). Among the 9 patients with resected benign lesions, there were 7 false-positive PET and 2 true-negative results. In the remaining 4 patients with benign disease, lung resection was avoided based on the negative PET results. The overall sensitivity with respect to pulmonary metastases was then $87 \%$, specificity $46 \%$, positive predictive value $87 \%$, negative predictive value $46 \%$.

Table 4 describes the analysis of SUV data in relation to pathologic characteristics. Mean SUV was higher in patients with colon cancer than in those with sarcoma, but no significant difference was evident between these 2 tumor types. A borderline $P$ value was demonstrated when comparing SUV of benign and malignant lesions (.08), but it increased to .14 when adjusting SUV for nodule size. Mean SUV was lower in the 7 patients who had received preoperative chemotherapy within 3 months of lung metastasectomy $(2.7 \pm 2.2$ vs $4.5 \pm 3.5, P=.2)$, but SUV reduction did not affect sensitivity, which remained $100 \%$ in this subset of patients.

\section{Discussion}

On the basis of CT scan staging, lung metastasectomy can achieve permanent cure and long-term survival in over $30 \%$ of cases. ${ }^{1-4}$ Presence of extrathoracic deposits in patients with lung metastasis is the main reason for exclusion from salvage surgery, whereas only recent reports have emphasized the importance of mediastinal staging in lung metastasis, indicating poor prognosis if nodal metastases are present. ${ }^{3,9,10}$

The main result of our study was the high frequency of positive extrapulmonary PET foci. This observation has modified the treatment plan in over $20 \%$ of patients. PET detection of occult distant metastases or recurrence at the primary site avoided unnecessary lung resections or
TABLE 4. Histology of lung lesions and SUV

\begin{tabular}{lccccc}
\hline Histology & $\begin{array}{c}\text { No. of } \\
\text { patients }\end{array}$ & $\begin{array}{c}\text { No. of } \\
\text { lesions* }\end{array}$ & $\begin{array}{c}\text { Mean } \\
\text { SUV }\end{array}$ & $\begin{array}{c}\text { ISEM } \\
\text { Sung metasases }\end{array}$ & $\begin{array}{c}\text { Median } \\
\text { SUV }\end{array}$ \\
\hline Colorectal & 20 & 53 & 4.3 & 0.5 & 3.4 \\
Sarcoma & 7 & 28 & 4.3 & 0.7 & 3.5 \\
Urologic & 5 & 7 & 3.7 & 1.4 & 2.7 \\
Breast & 2 & 2 & 5.5 & 1.4 & 3.4 \\
Head/neck & 1 & 3 & 1.2 & 4.2 & 4.2 \\
Gynecologic & 2 & 3 & 6.6 & 0.2 & 1.2 \\
Thymus & 3 & 3 & 4.0 & 0.6 & 8.4 \\
Second primary & 5 & 5 & 3.1 & 0.8 & 3.7 \\
Carcinoid & 2 & 2 & 1.7 & 0.1 & 1.7 \\
Squamous & 1 & 1 & 2.8 & NA & 2.8 \\
Adenoca & 1 & 1 & 1.7 & NA & 1.7 \\
Sclc & 1 & 1 & 7.3 & NA & 7.3 \\
Benign & 5 & 5 & 2.2 & 1.1 & 1.5 \\
\hline
\end{tabular}

Malignant versus benign: $P=0.085$; colorectal versus sarcoma: $P=0.713$. *Only resected lesions with SUV data and pathological size available were considered for analysis.

changed therapeutic strategy with prior treatment of extrathoracic disease such as combined staged liver and lung metastasectomy. Sensitivity and specificity for detection of extrapulmonary deposits in patients with lung metastases were comparable with those reported by Pieterman and colleagues $^{5}$ in potentially resectable lung cancer $(85 \%$ and $90 \%$ vs $82 \%$ and $93 \%$, respectively). PET false-negatives were limited to a brain meningioma, a small breast cancer, and a pleural metastasis. In the present study PET was not able to downstage patients by excluding equivocal evidence of distant metastasis on initial CT staging.

In this series all mediastinal node metastases were detected by PET with a sensitivity and accuracy greater than contrast CT scan. Preoperative knowledge of mediastinal node involvement may allow a better management of these patients, as it is an indication for preoperative chemotherapy and radical lymphadenectomy. On the other hand, the knowledge on hilar node metastasis may be of great importance in planning an anatomic resection, such as segmentectomy or lobectomy, instead of a simple wedge or precision resection.

In our series, mediastinal node involvement was observed in $14 \%$ of patients with resected lung metastasis, which reproduces exactly the data reported by Loehe and colleagues. ${ }^{9}$ In addition, our study confirmed the high negative predictive value of PET in mediastinal staging, similar to that reported in lung cancer (100\% present study vs $95 \%$ Pieterman and colleagues ${ }^{5}$.) On the other hand, given the low positive predictive value on mediastinal staging (78\%), a confirmation with surgical mediastinoscopy or transbronchial biopsy is still necessary before excluding patients from surgery in the case of positive PET at the mediastinal level. Although mediastinal node metastases may have a lesser 
prognostic relevance in thyroid or kidney cancer, this was not the case of the 2 patients excluded from surgery in our series (sarcoma and bladder cancer). Sensitivity of PET for detection of hilar nodes was only $40 \%$, but it was higher than CT scan. If we consider the contribution of PET scan on nodal staging, the overall change in patient management could be higher than $30 \%$ of cases.

With the increasing sensitivity of spiral CT the main limitation of radiologic assessment will become the differential diagnosis of small undetermined pulmonary nodules in patients with previous malignancy, rather than presence of occult lung metastasis. Lucas and colleagues ${ }^{11}$ reported an $86 \%$ sensitivity rate of FDG-PET in detection of lung metastases from soft tissue tumors and a sensitivity of $100 \%$ of chest CT. Lowe and colleagues ${ }^{12}$ reported sensitivity and specificity rates of $92 \%$ and $90 \%$, respectively, in identifying malignant features in undetermined lung nodules in patients with or without previous malignancy. In the present study, the overall PET sensitivity on lung metastasis was $87 \%$ and the capability of a noninvasive method to differentiate benign from malignant nodules avoided biopsy in 4 cases. On the other hand, the high rate of false-positive PET caused by chronic inflammation or infection and the low specificity and negative predictive value (46\%) still represent a limitation of this technique. ${ }^{13}$ Our results, in disagreement with previous observations,,${ }^{12}$ could be partially attributable to the absence of a cutoff value of SUV to differentiate benign from malignant nodules.

A significantly lower sensitivity was evident for nodules of less that $1 \mathrm{~cm}$ (40\% vs $90 \%)$. PET tended to be more sensitive in the case of colon cancer lesions than in sarcomas, but the difference was not statistically significant. In agreement with prior reports, PET accuracy was similar to that of spiral CT in detection of lung nodules ${ }^{11,14}$ and the use of PET did not supply any additional information to alter the surgical approach (ie, bilateral vs monolateral).

Analysis of SUV in different tumor types has shown higher value of SUV in colon cancer metastasis than in sarcomas (not significant). Comparison of mean SUV value in malignant nodules and benign ones demonstrated a borderline $P$ value, which became clearly not significant when adjusting SUV data for nodule size.
In conclusion our experience demonstrates that PET can improve the management of resectable lung metastases in a high proportion of patients. Our findings support the important role of PET in the selection of surgical candidates for lung metastasectomy with the aims of improving detection of extrathoracic disease, identifying nodal metastases, and increasing the accuracy of differential diagnosis of benign from malignant lung lesions.

\section{References}

1. Rusch VW. Pulmonary metastasectomy. Current indication. Chest. 1995; 107:S322-31.

2. Pastorino U. History of the surgical management of pulmonary metastases and development of the International Registry. Semin Thorac Cardiovasc Surg. 2002;14:18-28.

3. Girard P, Baldeyrou P, Le Chevalier T, Le Cesne A, Brigandi A, Grunenwald D. Surgery for pulmonary metastases. Who are the 10year survivors? Cancer. 1994;74:2791-7.

4. Pastorino U, Buyse M, Friedel G, et al. Long-term results of lung metastasectomy: prognostic analyses based on 5206 cases. The International Registry of Lung Metastases. J Thorac Cardiovasc Surg. 1997;113:37-49.

5. Pieterman RM, van Putten JWG, Meuzelaar JJ, et al. Preoperative staging of non-small-cell lung cancer with positron emission tomography. $N$ Engl J Med. 2000;343:254-61.

6. Saunders C, Dussek JE, O'Doherty MJ, Maisey MN. Evaluation of fluorine-18-fluorodeoxyglucose whole body positron emission tomography in the staging of lung cancer. Ann Thorac Surg. 1999;67:790-7.

7. Gupta NC, Graeber GM, Bishop HA. Comparative efficacy of positron emission tomography with fluorodeoxyglucose in evaluation of small $(<1 \mathrm{~cm})$, intermediate $(1$ to $3 \mathrm{~cm})$ and large $(>3 \mathrm{~cm})$ lymph node lesions. Chest. 2000;117:773-8.

8. Kalff VV, Hicks RJ, MacManus P, et al. Clinical impact of (18) F fluorodeoxyglucose positron emission tomography in patients with non-small-cell-lung cancer: a prospective study. J Clin Oncol. 2001; 19:111-8.

9. Loehe F, Kobinger S, Hatz RA, Helmberger T, Lohers U, Fuerst H. Value of systematic mediastinal lymph node dissection during pulmonary metastasectomy. Ann Thorac Surg. 2001;72:225-9.

10. Okumura S, Kondo H, Tsuboi M, et al. Pulmonary resection for metastatic colorectal cancer: experiences with 159 patients. J Thorac Cardiovasc Surg. 1996;112:867-74.

11. Lucas JD, Doherty MJ, Wong JCH, et al. Evaluation of fluorodeoxyglucose positron emission tomography in the management of softtissue sarcomas. J Bone Joint Surg. 1998;80B:441-7.

12. Lowe VJ, Fletchetr JW, Gobar L, et al. Prospective investigation of positron emission tomography in lung nodules. J Clin Onc. 1998;16: 1075-84.

13. Coleman RE. PET in lung cancer. J Nucl Med. 1999;40:818-20.

14. Kutlu CA, Pastorino U, Maisey M, Goldstraw P. Early experience with PET scanning in thoracic tumors. J Cardiovasc Surg. 2001;42:403-10. 